\title{
Régime alimentaire de quatre espèces du genre Haplochromis (Teleostéi, Cichlidae) du lac Ihema (Rwanda)
}

\author{
Y. Fourniret ${ }^{1}$ \\ P.-D. Plisnier ${ }^{1}$ \\ J.C. Micha1
}

Mots clés : Haplochromis, Gaurochromis, Paralabidachromis, Harpagochromis, régime alimentaire, écologie, lac Ihema, Rwanda.

L'étude comparée du régime alimentaire et du rythme d'activité de 4 espèces du genre Haplochromis, représentant, au lac Ihema, $27,9 \%$ du poids total des captures par pêches expérimentales montre que chacune présente une niche écologique distincte permettant d'éviter une compétition trophique importante. Ainsi Haplochromis (Haplochromis) sp. se nourrit, en zone pélagique, de larves de Chaoboridae près de la surface et pendant la nuit. Ces larves sont aussi ingèrées au large par $H$. (Gaurochromis) sp, mais plutôt pendant la journée et dans la vase du fond du lac. H. (Paralabidochromis) sp. est un benthogphage spécialisé dans la consommation des Chironomidae et des Oligochètes en zone côtière. Enfin, H. (Harpagochromis) sp. est un ichtyophage confirmé.

Feeding habits of four species belonging to the genus Haplochromis (Teleostei, Cichlidae) in Ihema lake (Rwanda)

Keywords : Haplochromis, Gaurochromis, Paralabidochromis, Harpagochromis, food, ecology, lake Ihema, Rwanda.

The food of four haplochromine species is investigated in this study. These species represent $27,9 \%$ of the total weight caught by experimental fishing. This work shows that each species takes advantage of specific food items. Haplochromis (Haplochromis) sp. feeds mainly on Chaoboridae, during the night, near the surface. $H$. (Gaurochromis) sp. is also an insect feeder whose main prey are Chaoboridae, but during the day and on the bottom. H. (Harpagochromis) sp. is a predator feeding mainly on fishes. The food of $H$. (Paralabidochromis) sp. is composed of benthic organisms, especially Chironomidae and Oligochaetes in the coastal zone. This high specialisation seems an efficient way to avoid food competition.

\section{Introduction}

Au Rwanda, pays où la démographie est galopante, le régime alimentaire de la population humaine est carencé en protéines animales. C'est pourquoi, déjà en 1968, la commission mixte belgorwandaise de coopération entre les deux pays recommandait qu'une étude hydrobiologique des lacs du Parc National de l'Akagera, et plus particulièrement du lac Ihema, soit entreprise en vue d'examiner leurs possibilités d'exploitation économique.

Suite à une mission d'identification menée par Micha en 1975, l'exploitation du lac commence en

1. Unité d'Ecologie des Eaux Douces, Facultés Universitaires Notre-Dame de la Paix, Rue de Bruxelles, 61 B-5000 Namur, Belgique.
1980 et est accompagnée, en parallèle, d'études sur l'évaluation des ressources piscicoles du lac et sur la biologie des espèces (Plisnier 1990, Plisnier et al. 1988).

Actuellement, la pêcherie du lac Ihema exploite environ 180 tonnes par an de Tilapia (Oreochromis niloticus, O. macrochir, Tilapia rendalli) et Clarias gariepinus. (Plisnier et al. 1988). Or, des pêches expérimentales récentes faites à l'aide de 10 filets maillants monofilaments de 8 à $60 \mathrm{~mm}$ entre-nœuds montrent que ces taxons ne représentent respectivement que $15,2 \%$ et $4,7 \%$ du poids total capturé alors que les captures de groupe des Haplochromis spp sont bien plus élevées et atteignent $27,9 \%$ du poids total des captures. L'exploitation rationnelle de ces Haplochromis sp. devrait permettre d'augmenter la production de la pêcherie Ihema et d'améliorer sa rentabilité. Cela serait également possible 
dans une série d'autres lacs du Rwanda où les Haplochromis sont particulièrement abondants (Micha \& Frank 1991).

Toutefois, préalablement à toute exploitation, il importe de préciser les caractéristiques biologiques essentielles des espèces à exploiter, alimentation, reproduction, dynamique de population... Les connaissances actuelles sur la biologie des Haplochromis spp. du Rwanda sont très faibles, sinon totalement inexistantes pour la majorité des espèces à l'exception de celles du lac Kivu (Ulyel et al. 1990). Des travaux en cours permettront de mieux cerner les principales caractéristiques tant en ce qui concerne la systématique des Haplochromis spp. du Rwanda (Snoeks, com. pers.) que la biologie des Haplochromis spp. des lac Ihema et Muhazi. Ce document expose et compare les premiers résultats de l'analyse du régime alimentaire de 4 espèces d' $H a$ plochromis du lac Ihema.

Le genre Haplochromis Hilgendorf, 1888, est essentiellement représenté au lac Ihema par au moins quatre espèces probablement nouvelles mais non encore décrites formellement et qui se différencient d'emblée par le fait qu'elles appartiennent à quatre sous-genres : H. (Haplochromis), H. (Gaurochromis), H. (Harpagochromis) et $H$. (Paralabidochromis). Ces derniers ont été élevés au rang de genre par Greenwood $(1979,1980)$. II faut noter cependant que cette classification est contestée par divers systématiciens (Thys \& Snoeks, com. pers., Barel \& Witte, com. pers. ; Teugels in Daget et al. 1991) mais nous conserverons ici la classification précédemment utilisée qui permet, dans le cas du lac Ihema, de bien distinguer ces taxons.

Enfin, il faut signaler que ces espèces appartenant à différents sous-genres sont probablement des cspèces nouvelles. Elles sont en voie de description par les systématiciens (Snoeks, com. pers.) du Musée Royal d'Afrique Centrale de Tervuren. Les spéciments de Haplochromis (Haplochromis) sp. sont référencés sous le $n^{\circ}$ MRAC 8208-359, ceux de $H$. (Gaurochromis) sp. sous le $\mathbf{n}^{\circ}$ MRAC 172289, ceux de $H$. (Harpagochromis) sp. sous le $n^{\circ}$ MRAC 164817 et ceux de $H$. (Paralabidochromis) sp. sous le $n^{\circ} \mathrm{MRAC} 164826$.

\section{Le milieu}

Situé à l'Est du Rwanda, à $1^{\circ} \mathbf{4 2}$ '42" au sud de l'Equateur, le lac Ihema (Fig. 1) est le plus grand des lacs de la zone marécageuse du parc de l'Akagera. Il couvre une superficie d'environ $90 \mathrm{~km}^{2}$ à une altitude de 1291 mètres. Ce lac de plaine inondée présente une profondeur moyenne de 4 à 5 mètres. Son niveau varie selon celui de la rivière Akagera qui l'alimente presqu'exclusivement.

Les précipitations locales sont faibles (700 à $800 \mathrm{~mm} / \mathrm{an}$ ) et les températures relativement élevées $\left(25^{\circ} \mathrm{C}\right.$ de température moyenne annuelle).

Il est important de noter, pour la faune piscicole, que les eaux superficielles ne sont jamais dépourvues d'oxygène : la sursaturation est quotidienne, allant jusqu'à $130 \%$. Par contre, en profondeur, un dćficit dc $\mathrm{O}_{2}$ pcut apparaître durant la saison sèche, causant une stratification, cependant toujours fort labile.

Pauvre en électrolytes, sa conductivité est faible $\left(90-145{ }_{\mu} \mathrm{S} / \mathrm{cm}\right)$ avec une carence en azote et une absence de sulfate. Ceci résulte du bassin versant où dominent les quartz et les schistes.

La faible transparence de l'eau (environ $70 \mathrm{~cm}$ ) est due à la richesse du lac en phytoplancton. La chlorophylie a, mesurée en septembre 1984, présente une concentration moyenne de $70 \mathrm{mg} / \mathrm{m}^{3}$ (Descy \& Theate, com. pers.).

Etant un lac de plateau à maturité eutrophe, il est classé dans la catégorie des lacs tropicaux à faible profondeur, holomictiques, monomictiques chauds, présentant une eau asulfatée (Kiss 1976).

\section{Matériel et méthodes}

\subsection{Filets et zones de pêche}

Les échantillons d'Haplochromis spp. ont été récoltés du 25 janvier 1988 au 22 avril 1988 pour la plupart des espèces et du 15 mars 1988 au 31 août 1988 pour $H$. (Gaurochromis) sp. Les captures sont effectuées à l'aide de 6 filets maillants de 8,10 et $20 \mathrm{~mm}$ d'entrenceuds de $25 \mathrm{~m}$ de long pour $2 \mathrm{~m}$ de hauteur. Une batterie de filets pêche sur le fond (ralingue fortement plombée : $\mathbf{3 2} \mathrm{g} / \mathrm{m}$ ), l'autre en surface (ralingue faiblement plombee : $8 \mathrm{~g} / \mathrm{m}$ ).

Afin d'obtenir des contenus stomacaux fraîchement ingérés et non encore trop attaqués par les enzymes digestifs, ainsi que pour suivre le rythme nychténémal de ces espèces, nous avons effectué 6 cycles de 24 heures de pêche en zone pélagique. Les filets étaient relevés toutes les 3 heures. Immédiatement après la relève des filets, le nombre de 


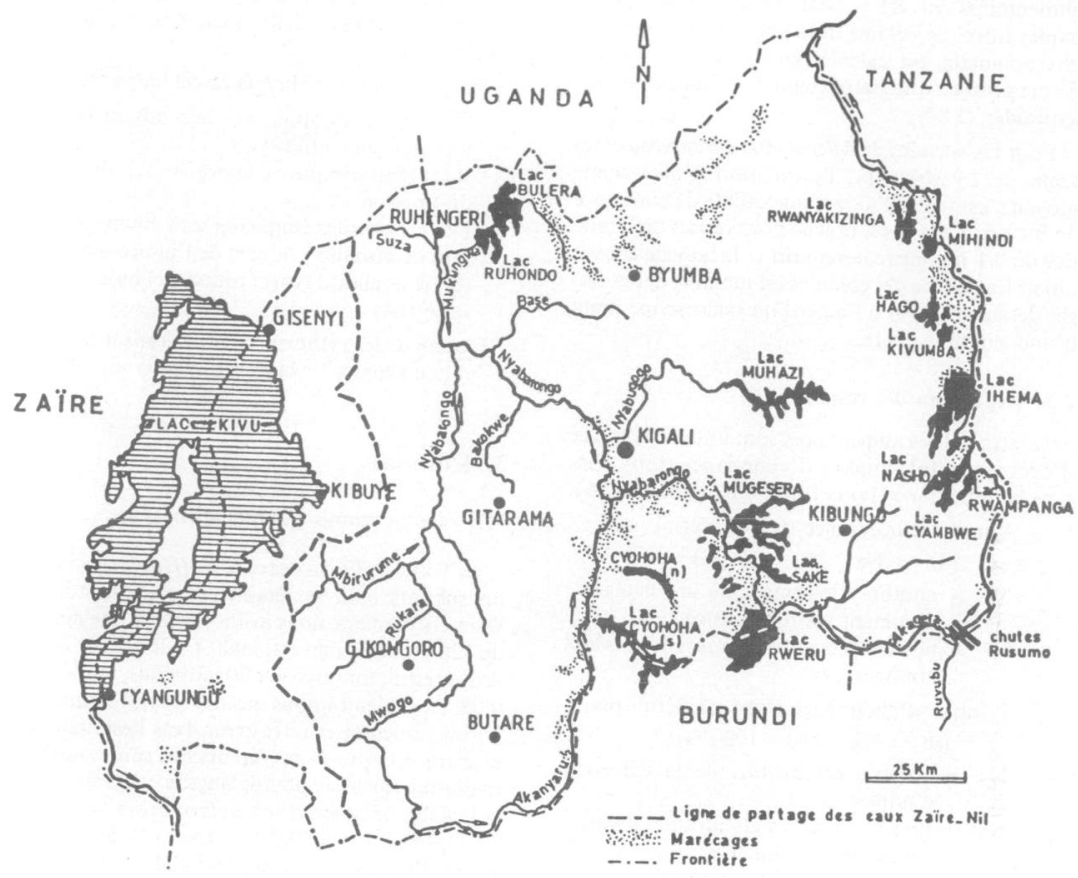

Fig. 1. Situation générale et réseau hydrographique du Rwanda appartenant au bassin du Nil.

Fig. General plan and hydrographic system of the Rwanda region of the Nile basin.

poissons de chaque taxon capturé par les différents filets est noté. Ensuite, les poissons sont disséqués. Chaque poisson est identifié, pesé, mesuré (longueur totale et longueur standard en $\mathrm{mm}$ ) et sexé. Les estomacs jugés suffisamment remplis sont conservés au formol à $7 \%$. Un coefficient de réplétion de l'estomac est attribué et le contenu de celui-ci est pesé. Pour 20 individus de chaque espèce, la longueur de l'intestin a été mesurée au millimètre près et, pour quelques poissons, l'intestin fut prélevé pour une analyse succincte du contenu.

\subsection{Méthodes d'analyse}

Un premier tri, lors de l'ouverture des estomacs, a permis d'éliminer les contenus trop dégradés. Seuls ont été analysés les contenus stomacaux en bon état, présentant des proies identifiables. Les différents éléments sont séparés, déterminés et comptés. Leur volume est évalué par déplacement d'eau à l'aide de pipettes graduées de différentes tailles selon le volume des proies et précises jusqu'à $0,01 \mathrm{ml}$. Avant d'être plongé dans l'eau des pipettes, les constituants 
alimentaires ont été préalablement séchés sur du papier filtre. Le volume de la plupart des taxons du phytoplancton est calculé par assimilation à une forme géométrique simple selon la méthode de Vollenweider (1969).

Pour les colonies de Microcystis aeruginosa et les amas de Lyngbya sp., l'estimation d'un volume moyen s'est faite à l'aide d'une cellule de comptage de Burker. Celle-ci est prévue pour laisser un interstice de $0,1 \mathrm{~mm}$ entre le support et la lamelle couvre objet. La surface des colonies est mesurée après dessin de leur contour à l'aide d'un microscope muni d'une chambre claire.

\subsection{Expression des résultats}

Quatre indices alimentaires sont utilisés : I'indice d'occurence (Io), l'indice d'abondance (Iab), l'indice volumétrique (Iv) et l'indice alimentaire (IA).

- L'indice d'occurence (lo) est défini par :

$$
\mathrm{Io}=\mathrm{Na} / \mathrm{Nt} \cdot 100(\%)
$$

$\mathrm{Na}=$ nombre d'estomacs où une catégorie d'aliment est représentée

$\mathrm{Nt}=$ nombre total d'estomacs non vides analysés

- L'indice d'abondance (Iab) est défini par :

$$
\text { lab }=N_{x} / N_{x t} .100(\%)
$$

$\mathrm{Nx}=$ nombre d'individus de la catégorie d'aliment $x$

Nxt = nombre total d'individus des diverses catégories d'aliment $\mathrm{x}$

- L'indice volumétrique (Iv) est défini par :

Iv $=(\mathrm{Vx} . \mathrm{Nx}) / \mathrm{Vt} .100(\%)$

$\mathrm{Vx}=$ volume individuel moyen de chaque catégorie (en $\mathrm{ml}$ )

$\mathrm{Vt}=$ volume total des aliments ingérés (en $\mathrm{ml}$ )

Le quatrième indice est l'indice alimentaire défini par Lauzanne (1975 et 1976). Celui-ci utilise les méthodes volumétriques (Iv) et les pourcentages d'occurence (lo). Cet indice se calcule comme suit :

$$
\mathrm{IA}=(\mathrm{Iv} . \mathrm{Io}) / 100
$$

IA = indice alimentaire de Lauzanne (compris entre 0 et 100 )

Iv = pourcentage volumétrique $(\%)$.

Io $=$ pourcentage $\mathrm{d}^{3}$ occurence $(\%)$.

Il est possible de cette manière de classer les proies en quatre catégories (IA inférieur à 10: proie secondaire, entre 10 et 24 : proic importante, entre 25 et 50 : proie essentielle et entre 50 et 100 : proie dominante).

Nous pensons que l'intérêt de cet indice est triple :

- Des méthodes objectives minimisant le risque d'erreur sont utilisées.

- Il est tenu compte de la préférence alimentaire du poisson.

- Des graphiques éloquents sont établis, permettant de visualiser la part de l'indice quantitatif (iv) et qualitatif (Io) constituant l'indice alimentaire (IA).

L'analyse des rythmes nychtéméraux a été réalisé à l'aide du logiciel Maxims (Jarre et al. 1990).

\section{Résultats}

\subsection{Haplochromis (Haplochromis) sp.}

Un total de 89 estomacs d' $H$. (Haplochromis) sp. ont subi une analyse détaillée du contenu stomacal. Dans ces derniers, nous avons identifié une moyenne de $\mathbf{2 2 6 , 6}$ proies par estomac. La longueur relative de l'intestin, mesurée sur 20 individus, est de $1,7 \pm$ 0,09 . Le tableau I nous montre les différentes catégories alimentaires retrouvées dans l'estomac de ce poisson. Les proies préférentielles sont constituées majoritairement de phytoplaneton : une algue bleue coloniale, Microcystis aeruginosa (lab $=78,1 ; \mathrm{lv}$ $=72,9 \%$; Io $=69,7 \%$; IA $=50,8$ ) et par des larves d'insectes Chaoboridae (lab $=17,3 \%$; Iv $=22,7 \% ; \mathrm{lo}=82,0 \% ; \mathrm{IA}=18,7)$. Le spectre alimentaire représenté pour les 3 catégories alimentaires majoritaires montre bien la dominance des Microcystis aeruginosa suivis par les larves de Chaoboridae (Fig. 2). Les autres proies sont accidentelles et, exceptées les pupes de Chaoboridae, ne représentent qu'un apport énergétique infime.

Bien que l'indice volumétrique du phytoplancton des deux classes de tailles de IIaplochromis (Haplochromis) sp. soit similaire, l'occurrence de celui-ci est réduite de $50 \%$ chez les plus grands individus (Io $=40,1 \%$ ) (Tableau II). Les individus plus grands semblent donc marquer moins de préférence pour le phytoplancton.

On constate très peu de différence dans le régime alimentaire des mâles et des femelles de H. (Haplochromis) sp. (Tableau III). 
Le rythme nycthéméral de prise alimentaire de cette espèce est représenté à la figure 3. $H$. (Haplochromis) sp. présente deux périodes d'alimentation. La première commence à la fin de la nuit, s'arrête peu après l'aube, vers 8 heures, et la seconde, moins intense, débute dès le début de la nuit pour s'interrompre à 22 heures. Reflet de son activité locomotrice, les captures par tranche horaire, provenant des filets maillants, indiquent qu'H. (Haplochromis) sp. débute ses déplacements vers 3 heures du matin. Son activité est intense curant la matinée (6 à 12 heures) puis baisse pour reprendre à 15 heures et culminer une seconde fois entre 18 et 21 heures.

\subsection{H. (Gaurochromis) sp.}

Les échantillons analysés proviennent de $97 \mathrm{H}$. (Gaurochromis) sp. de longueur totale comprise entre 58 et $140 \mathrm{~mm}$. La longueur relative de l'intestin, mesurée sur 8 individus, est de $1,97 \pm 0,39$.
H. (Gaurochromis) sp. se nourrit principalement d'insectes ( $\mathrm{V} v=94,6 \%$ ). Il s'agit presqu'exclusivement de diptères du genre Chaoborus consommés sous forme de larves (Io $=56,7 \%, \mathrm{Iv}=69,7 \%$ ) et de pupes ( $\mathrm{lo}=43,3 \%$, Iv $=23,9 \%$ ) (Tableau I). Le spectre alimentaire des 3 catégories dominantes (fig. 2) illustre parfaitement la specialisation des H. (Gaurochromis) sp. pour la consommation des larves et pupes d'insectes Chaoboridae.

Toutefois, il faut signaler que du phytoplancton (Diatomées, Cyanophycées) a été observé fréquemment dans les contenus stomacaux : Melosira $(\mathrm{Io}=$ $30,9 \%)$, Lyngbia sp (Io $=22,7 \%$ ) et Microcystis aeruginosa $(\mathrm{Io}=21,6 \%)$.

L'indice d'abondance est le plus élevé pour le phytoplancton $(95,6 \%)$, ce qui est dû en grande partie à des amas très importants de Lyngbia sp. dans plusieurs estomacs. Il faut encore remarquer la présence de débris de macrophytes $(\mathrm{Io}=35,1 \%$ ) et de copépodes $(\mathrm{Io}=13,4 \%$ ) dans les estomacs.

Tableau 1. Indice d'occurrence (Io), indice d'abondance (Iab), indice volunétrique (Iv) et indice alimentaire ([A) d'H. (Haplochromis) sp. (n $=89), H$. (Gaurochromis) sp. $(\mathrm{n}=97), H$. (Harpagochromis) sp. $(\mathrm{n}=70)$ et $H$. (Paralabidochromis) sp. (n $=30)$ au lac lhema, tailles et sexes confondus (" $P<0,1$ ).

Table 1. Indices of occurence (lo), abundance (Iab), volume (Iv) and feeding (IA) of H. (Haplochromis) sp. (n $=89$ ), H. (Gaurochromis) sp. $(\mathrm{n}=97), H$. (Harpagochromis) sp. $(\mathrm{n}=70)$ et $H$. (Paralabidochromis) sp. $(\mathrm{n}=30)$ from Ihema lake, different sizes and sexes mixed $(* P<0.1)$.

\begin{tabular}{|c|c|c|c|c|c|c|c|c|c|c|c|c|c|c|c|c|}
\hline & \multicolumn{4}{|c|}{ H. (Haplochromis) $\Phi$} & \multicolumn{4}{|c|}{ H. (Gawrochromis) sp } & \multicolumn{4}{|c|}{ H. (Harpogachromis) sp } & \multicolumn{4}{|c|}{$\overline{H . ~(P a r a l a b i d a c h r o m i s) ~ s p ~}$} \\
\hline & 10 & Iab & Iv & iA & lo & $1 a b$ & Iv & IA & Io & $\mathrm{Iab}$ & Iv & IA & Io & lab & Iv & IA \\
\hline Insectes & & & & & & 0,1 & 94,6 & 26,8 & & & & & & & & \\
\hline Larves de Chaoborus & 82,0 & 17,4 & 22,7 & 18,7 & 56,7 & $\bullet$ & 69.7 & 39,5 & 14,3 & 10,2 & 0,2 & * & 33,3 & 28,8 & 11,4 & 3,8 \\
\hline Pupes de Chaoborus & 27.5 & 2,8 & 4,2 & 1,2 & 43,3 & - & 23,9 & 10,4 & 10,0 & 8,3 & 0,3 & * & & & & \\
\hline Larves de Chironomidae & & & & & 5.2 & - & 1.0 & 0.1 & & & & & 66,7 & 30,5 & 36,4 & 24,2 \\
\hline Nymphe de Chironomidac & & & & & & & & & & & & & 10.0 & 1.2 & 1,9 & 0.2 \\
\hline Ephèméroptżtes & & & & & & & & & 2,9 & $0, \dot{2}$ & 0,1 & * & 6.7 & 0,2 & & \\
\hline Noctonectidac & 1,1 & - & 0.1 & - & & & & & 7,1 & 0,9 & 0,2 & * & 3.3 & 0.1 & * & * \\
\hline Trichopieres & 1,1 & - & & & & & & & & & & & & & & \\
\hline Diptkes non id. & 45 & $\bullet$ & & & & & & & & & & & 6.7 & 0,2 & & \\
\hline Insectes non id. & & & & & 8.2 & & & & & & & & & & & \\
\hline Zooplancton & & & & & 3.9 & $*$ & $\bullet$ & * & & & & & & & & \\
\hline Cindocères & 4,5 & 0,4 & - & - & 0.0 & 0,0 & 0,0 & 0.0 & & & & & & & & \\
\hline Copépodes & 12,3 & 3,3 & • & - & 13.4 & $*$ & $\bullet$ & $*$ & & & & & 13.3 & 0,9 & $*$ & - \\
\hline Ostracodes & & & & & 0,0 & 0,0 & 0,0 & 0,0 & & & & & 40,0 & 18,0 & 0,2 & 0.1 \\
\hline Rotiferes & & & & & 2.1 & $\bullet$ & $\bullet$ & $*$ & & & & & & & & \\
\hline Phytoplancton & 69,7 & 78,1 & 72,9 & 50.8 & 6.7 & 95.6 & $\$ .4$ & 0,4 & 5.7 & 75.2 & 1,2 & 0,1 & 13,3 & 12.9 & 3.7 & 0.5 \\
\hline Poissons (restes) & & & & & & 0,0 & & & 5.7 & 0,4 & 3.1 & 0.2 & & & & \\
\hline Haplochrornis spp & & & & & & & & & 48,6 & 3.2 & 82.5 & 40,0 & & & & \\
\hline Tilapia spp & & & & & & & & & 8,6 & 0,5 & 4,4 & 0,4 & & & & \\
\hline Deufs & & & & & & & & & 1,4 & 0,2 & 7,6 & 0,1 & & & & \\
\hline Alevins & & & & & & & & & & & & & 13,3 & 0,7 & 40,0 & 5,3 \\
\hline Ecrailles & & & & & & & & & 5.7 & & & & & & & \\
\hline Macrophytes & & & & & 35,1 & 2,8 & & & 7,1 & & & & 63,3 & & & \\
\hline Crustacts & & & & & & & & & 1,4 & 0.1 & 0,2 & * & & & & \\
\hline Mollusques & & & & & & & & & & & & & & & & \\
\hline OHgochtes & & & & & & & & & & & & & 43,3 & 5,9 & 6.4 & 2.8 \\
\hline Indtermint & & & & & 9,3 & 0.7 & & & 7,1 & 0,7 & 0,4 & • & 16.7 & 0.8 & & \\
\hline Sable & & & & & 10.3 & 0.8 & & & & & & & 50.0 & & & \\
\hline Towel & & 100 & 100 & & & 100 & 100 & & & 100 & 100 & & & 100 & 100 & \\
\hline
\end{tabular}


Tableau 2. Indice d'occurrence (lo), indice d'abondance (Iab), indice volu métrique (Iv) et indice alimentaire (IA) d'H. (Haplochromis) sp. $(\mathrm{n}=89)$, H. (Gaurochromis) sp. $(\mathrm{n}=97)$, H. (Harpagochromis) sp. $(\mathrm{n}=70)$ et H. (Paralabidochromis) sp. $(\mathrm{n}=30)$ du lac Ihema, répartis en fonction des classes de taille $(* P<0,1)$.

Table 2. Indices of occurrence (Io), abundance (lab), volume (Iv) and feeding (IA) of H. (Haplochromis) sp. (n $=89$ ), H. (Gaurochromis) sp. (n $=97)$. H. (Harpagochromis) sp. $(\mathrm{n}=70)$ et $H$. (Paralabidochromis) sp. ( $\mathrm{n}=30)$ from Ihema lake, fish separated into different size classes $(* \mathrm{P}<0.1)$.

\begin{tabular}{|c|c|c|c|c|c|c|c|c|c|c|c|c|c|c|c|c|}
\hline & \multicolumn{8}{|c|}{ H. (Haplochromis) sp I } & \multicolumn{8}{|c|}{ H. (Gaurochroem is) $\mathbf{s p}$} \\
\hline & \multicolumn{4}{|c|}{$54-65 \mathrm{~mm}(n=45)$} & \multicolumn{4}{|c|}{$66-85 \mathrm{~mm}(n=44)$} & \multicolumn{4}{|c|}{$58-88 \mathrm{~mm}(\mathrm{n}=43)$} & \multicolumn{4}{|c|}{$88-140 \mathrm{~mm}(n=54)$} \\
\hline & lo & $\mathrm{lab}$ & IV & LA & lo & Iab & Iv & LA & lo & lab & I & LA & Io & Inb & lv & IA \\
\hline Insectes & & 20,6 & 27,6 & & & 19,2 & 26,1 & & & 2,0 & 91.0 & & & 0,2 & 95.8 & \\
\hline Larves de Chaobonus & 82.2 & 19.4 & 25,6 & 21,0 & 79,6 & 13,5 & 17,5 & 13,9 & 37.2 & 0,1 & 82,6 & 30.7 & 72.2 & $\bullet$ & 65.1 & 47.0 \\
\hline Pupes de Chaobonus & 15.6 & 1.3 & 2,0 & 0,3 & 39,8 & 5,6 & 8,4 & 3,3 & 25.6 & $*$ & 8.3 & 2.1 & 57.4 & * & 29,4 & 16,9 \\
\hline Laves de Chironomidae & & & & & & & & & 0,0 & 0.0 & 0.0 & 0,0 & 9.3 & * & 1,3 & 0.1 \\
\hline \multicolumn{17}{|l|}{ Nymphe de Chironomidec } \\
\hline \multicolumn{17}{|l|}{ Ephèméroptères } \\
\hline Nactonectidne & 0.0 & 0,0 & 0,0 & 0,0 & 2,3 & + & 0,2 & + & & & & & & & & \\
\hline Trichoplères & 0,0 & 0.0 & 0,0 & 0,0 & 2,3 & • & & & & & & & & & & \\
\hline Diptères non id. & 2,3 & 0,0 & 0,0 & 0,0 & 2,3 & 0,1 & & & & & & & & & & \\
\hline Insectes non id. & & & & & & & & & 7,0 & 1,8 & & & 9,3 & & & \\
\hline Zooplancton & & 2,3 & 0.0 & & & 0,6 & 0.0 & & & - & 0,1 & & & • & • & \\
\hline Cladocères & 4,4 & 0,5 & - & - & 4.6 & 0,4 & $*$ & - & 0,0 & 0,0 & 0.0 & 0.0 & 0.0 & 0.0 & 0.0 & 0.0 \\
\hline Copépodes & 13.3 & 1,8 & $\star$ & - & 11,4 & 0.3 & • & - & 25.6 & 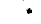 & 0.1 & 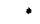 & 3,7 & 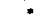 & • & $\bullet$ \\
\hline Ostracodes & & & & & & & & & 0,0 & 0,0 & 0.0 & 0,0 & 0.0 & 0.0 & 0,0 & 0.0 \\
\hline Rotiferes & & & & & & & & & 2,3 & 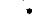 & * & * & 1.9 & * & • & $\bullet$ \\
\hline Phytoplancion & 80,0 & 77.1 & 72.5 & 58.0 & 40.1 & 80.1 & 73.9 & 29.6 & 5.0 & 88.4 & 9,0 & 0,5 & 8,1 & 96.7 & 4,2 & 0,3 \\
\hline Poissons (festes) & & & & & & & & & 0,0 & 0,0 & 0,0 & 0,0 & 0,0 & 0,0 & & \\
\hline \multicolumn{17}{|l|}{ Hapiochromis spp } \\
\hline \multicolumn{17}{|l|}{ Tilepia spp } \\
\hline \multicolumn{17}{|l|}{ Ocufs } \\
\hline \multicolumn{17}{|l|}{ Alevins } \\
\hline \multicolumn{17}{|l|}{ Ecailles } \\
\hline Macrophytes & & & & & & & & & 23,3 & 4,6 & & & 44,4 & 2,4 & & \\
\hline \multicolumn{17}{|l|}{ Crustaces } \\
\hline Mollusques & & & & & & & & & 0,0 & 0,0 & 0,0 & 0,0 & 0,0 & 0,0 & 0,0 & 0,0 \\
\hline \multicolumn{17}{|l|}{ Oligochètes } \\
\hline Indèlerminé & & & & & & & & & 14,0 & 2,7 & & & 5,6 & 0,3 & & \\
\hline Sable & & & & & & & & & 11,6 & 2,3 & & & 9,3 & 0,5 & & \\
\hline Total & & 100 & 100 & & & 100 & 100 & & & 100 & 100 & & & 100 & 100 & \\
\hline
\end{tabular}

\begin{tabular}{|c|c|c|c|c|c|c|c|c|c|c|c|c|c|c|c|c|}
\hline & \multicolumn{8}{|c|}{ W. (Harpagochromis) $\$$} & \multicolumn{8}{|c|}{ H. (Para labidochromis) sp } \\
\hline & \multicolumn{4}{|c|}{$68 \cdot 100 \mathrm{~mm} \quad(n=17)$} & \multicolumn{4}{|c|}{$106 \cdot 215 \mathrm{~mm}(\mathrm{n}=53)$} & \multicolumn{4}{|c|}{$68-85 \mathrm{~mm}(n=9)$} & \multicolumn{4}{|c|}{$88-128 \mathrm{~mm}(\mathrm{n}=21)$} \\
\hline & lo & lab & Iv & LA & lo & Iab & lv & IA & I0 & Iab & Iv & LA & lo & Iab & lv & IA \\
\hline Insectes & & 18,6 & 17,6 & & & 22,0 & 0,3 & & & 94.7 & 73,8 & & & 45,1 & 44,2 & \\
\hline Larves de Chaoburus & 41.2 & 8.3 & 5,3 & 2,2 & 5,7 & 14.5 & 0.1 & $\bullet$ & 55,6 & 87,3 & 59,3 & 32,9 & 23,8 & 1.4 & 0,5 & 0,1 \\
\hline Pupes de Chaoborus & 29,4 & 9,9 & 8.7 & 2,6 & 3.8 & 4.7 & $*$ & $\star$ & & & & & & & & \\
\hline Larves de Chironomidae & & & & & & & & & 33,3 & 7.1 & 14.5 & 4.8 & 81.0 & 41.5 & 41,4 & 33.5 \\
\hline Nymphe de Chironom idae & & & & & & & & & 0.0 & 0.0 & 0.0 & 0,0 & 14.3 & 1,7 & 2,3 & 0,3 \\
\hline Ephèmeroptères & 5,9 & 0,2 & 2,2 & 0,1 & 1,9 & 0,3 & $*$ & - & 0,0 & 0,0 & 0,0 & 0,0 & 9,5 & 0,2 & & \\
\hline Noctonectidae & 11,8 & 0.2 & 1.3 & 0,2 & 5,7 & 2,6 & 0.2 & $\bullet$ & 0,0 & 0,0 & 0,0 & 0,0 & 4,8 & 0.1 & * & - \\
\hline \multicolumn{17}{|l|}{ Trichoplères } \\
\hline Diptères non id. & & & & & & & & & 11,1 & 0,3 & & & 4,8 & 0.1 & & \\
\hline \multicolumn{17}{|l|}{ Lrsectes non id. } \\
\hline \multicolumn{17}{|l|}{ Zooplancton } \\
\hline \multicolumn{17}{|l|}{ Cladocères } \\
\hline \multicolumn{17}{|l|}{ Copépodes } \\
\hline Ostracodes & & & & & & & & & & & & & 57,1 & 26,4 & 0.3 & 0,2 \\
\hline \multicolumn{17}{|l|}{ Rotiferes } \\
\hline Phyloplancton & 17.6 & 80.9 & 37.0 & 6.5 & 1.9 & 62.2 & 0.3 & • & I1.1 & 4.9 & 2.4 & 0,3 & 14,3 & 16.7 & 4,0 & 0.6 \\
\hline Poissons (restes) & 0.0 & 0.0 & 0,0 & 0.0 & 7.6 & 13 & 3.1 & 0.2 & & & & & & & & \\
\hline Haplochuromis SPP & 5.9 & 0.3 & 10,3 & 0,6 & 62,3 & 10.4 & 84.2 & 52,0 & & & & & & & & \\
\hline Tilapia spp & 5,9 & 0.1 & 31,0 & 1,8 & 9,4 & 1.3 & 3,7 & 0.4 & & & & & & & & \\
\hline Deufs & 0,0 & 0,0 & 0.0 & 0.0 & 1,9 & 0,8 & 7,8 & 0.1 & & & & & & & & \\
\hline Alevins & & & & & & & & & 11,1 & 0,3 & 23,8 & 2,7 & 14,3 & 0.9 & 43,7 & 6.3 \\
\hline Ecailles & 18.0 & & & & 1,9 & & & & & & & & & & & \\
\hline Macrophytes & 12,0 & & & & 5.7 & & & & 22.2 & & & & 81.0 & & & \\
\hline Crustaces & 0.0 & 0,0 & 0.0 & 0,0 & 1,9 & 0.3 & 0,2 & $\bullet$ & & & & & & & & \\
\hline \multicolumn{17}{|l|}{ Mollusques } \\
\hline Oligochètes & & & & & & & & & 0,0 & 0.0 & 0,0 & 0.0 & 61,9 & 8.6 & 7.9 & 4.9 \\
\hline Indéterminé & 5.9 & 0,2 & 4.1 & 0.2 & 7.6 & 1.8 & 0.4 & $\bullet$ & 11.1 & 0.3 & & & 19.0 & 1.0 & & \\
\hline Sable & & & & & & & & & 11.1 & & & & 66.7 & & & \\
\hline Toual & & 100 & 100 & & & 100 & 100 & & & 100 & 100 & & & 100 & 100 & \\
\hline
\end{tabular}


Tableau 3. Indice d'occurrence (Io), indice d'abondance (Iab), indice volumétrique (Iv) et indice alimentaire (IA) d'H. (Haplochromis) sp. $(\mathrm{n}=89), H$. (Gaurochromis) sp. $(\mathrm{n}=97)$. H. (Harpagochromis) sp. $(\mathrm{n}=70)$ et H. (Paralabidochromis) sp. (n $=30)$ au lac thema, repartis en fonction des sexes $(* P<0,1)$.

Table 3. Indices of occurrence (Io), abundance (Iab), volume (Iv) and feeding (IA) of H. (Haplochromis) sp. (n = 89), H. (Gaurochromis) sp. (n = 97), H. (Harpagochromis) sp. $(\mathrm{n}=70)$ et $\boldsymbol{H}$. (Paralabidochromis) sp. (n $=30)$ from Ihema lake, fish separated into different sexes $(* P<0.1)$.

\begin{tabular}{|c|c|c|c|c|c|c|c|c|c|c|c|c|c|c|c|c|}
\hline & \multicolumn{8}{|c|}{ H. (Huplochroon is) sp 1} & \multicolumn{8}{|c|}{ H. (Gaurochromis) $\mathbf{s p}$} \\
\hline & \multirow[b]{2}{*}{10} & \multicolumn{3}{|c|}{ Mäles $(n=42)$} & \multicolumn{4}{|c|}{ Ferneles $(n=47)$} & \multicolumn{4}{|c|}{ Möles $(n=26)$} & \multicolumn{4}{|c|}{ Fernellest $(n=50)$} \\
\hline & & lab & Iv & IA & [o & Iab & Iv & IA & to & $\mathrm{fab}$ & lv & LA & Io & lab & Iv & IA \\
\hline Insectes & & 20,4 & 27,2 & & & 19,9 & 26.8 & & & 0,1 & 89,3 & & & 0,1 & 96,2 & \\
\hline Larves de Chaoborus & 90,5 & 19,5 & 26,0 & 23.5 & 74,5 & 14,9 & 19.1 & 14,3 & 53,8 & $\cdot$ & 35,7 & 19.2 & 68,0 & 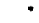 & 75,8 & 51,5 \\
\hline Pupes de Chaoborus & 23,8 & 0.9 & 1,3 & 0,3 & 30,9 & 5,0 & 7.5 & 2,3 & 57,7 & * & 52,7 & 30,4 & 42.0 & * & 19.2 & 8.1 \\
\hline Laves de Chironomidac & & & & & & & & & 3.8 & • & 1,0 & $\bullet$ & 8.0 & $\bullet$ & 1,3 & • \\
\hline \multicolumn{17}{|l|}{ Nymphe de Chironomidee } \\
\hline Ẹphèméroptères & & & & & & & & & & & & & & & & \\
\hline Noctonectidae & 0,0 & 0.0 & 0,0 & 0,0 & 2.1 & * & 0.1 & * & & & & & & & & \\
\hline Trichoptères & 0.0 & 0.0 & 0.0 & 0,0 & 2,1 & * & & & & & & & & & & \\
\hline Diptères non id. & 2,4 & * & & & 6,4 & 0.1 & & & & & & & & & & \\
\hline Insectes non id & & & & & & & & & 3.8 & & & & 12,0 & & & \\
\hline Zooplancton & & 2.9 & * & & & 0,3 & * & & & * & * & & & • & - & \\
\hline Oladocères & 2,4 & 0,6 & - & * & 6.4 & 0.3 & - & - & 0,0 & 0,0 & 0,0 & 0.0 & 0,0 & 0,0 & 0.0 & 0,0 \\
\hline Copépodes & 21.4 & 2,4 & 0,0 & 0.0 & 2.1 & * & - & $\cdot$ & 19.2 & 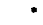 & $*$ & $\bullet$ & 10,0 & • & - & * \\
\hline Ostracodes & & & & & & & & & 0,0 & 0.0 & 0.0 & 0.0 & 0,0 & 0.0 & 0.0 & 0,0 \\
\hline Rotiferes & & & & & & & & & 3.8 & * & * & * & 2,0 & * & + & • \\
\hline Phytoplancion & 64,3 & 76,7 & 72.7 & 46,8 & 74,5 & 79,8 & 73,2 & 54,5 & 7,4 & 92,8 & 10,6 & 0,8 & 7,1 & 96,7 & 3,8 & 0,3 \\
\hline Puissons (resles) & & & & & & & & & & 0,0 & & & & 0,0 & & \\
\hline \multicolumn{17}{|l|}{ Haplochromis spp } \\
\hline \multicolumn{17}{|l|}{ Tilepin spp } \\
\hline \multicolumn{17}{|l|}{ Deufs } \\
\hline \multicolumn{17}{|l|}{ Alevins } \\
\hline \multicolumn{17}{|l|}{ Ecailles } \\
\hline Macrophytes & & & & & & & & & 30,8 & 3,8 & & & 38,0 & 2,3 & & \\
\hline \multicolumn{17}{|l|}{ Crustaces } \\
\hline Mollusques & & & & & & & & & 0,0 & 0,0 & 0,0 & 0.0 & 0,0 & 0,0 & 0,0 & 0,0 \\
\hline \multicolumn{17}{|l|}{ Oligochètes } \\
\hline Indélerminè & & & & & & & & & 15,4 & 1,9 & & & 4,0 & 0,2 & & \\
\hline Sable & & & & & & & & & 11.5 & 1.4 & & & 12,0 & 0,7 & & \\
\hline Tola] & & 100 & 100 & & & 100 & 100 & & & 100 & 100 & & & 100 & 100 & \\
\hline
\end{tabular}

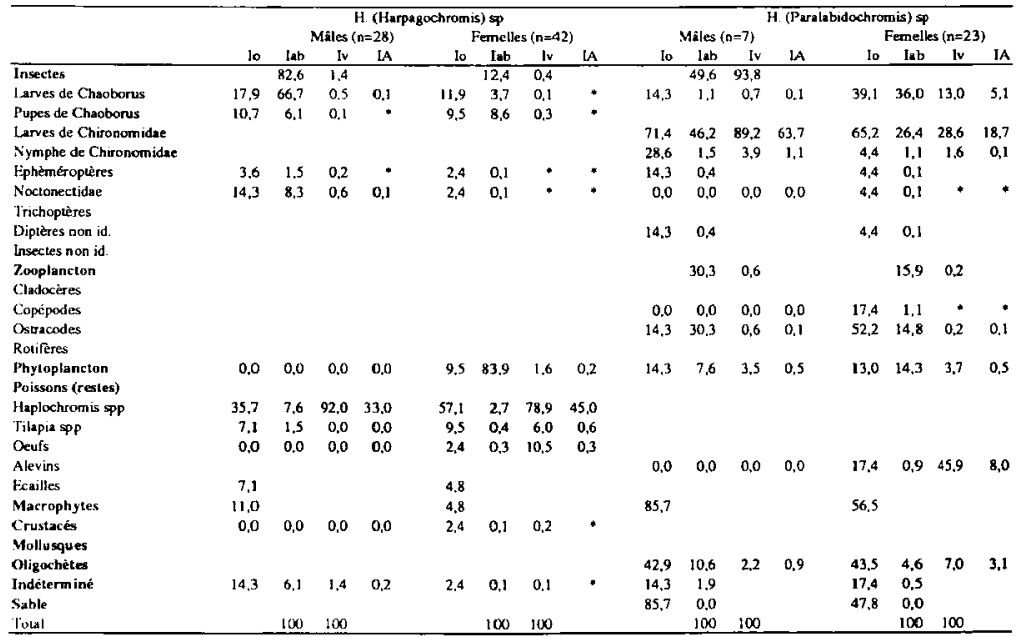



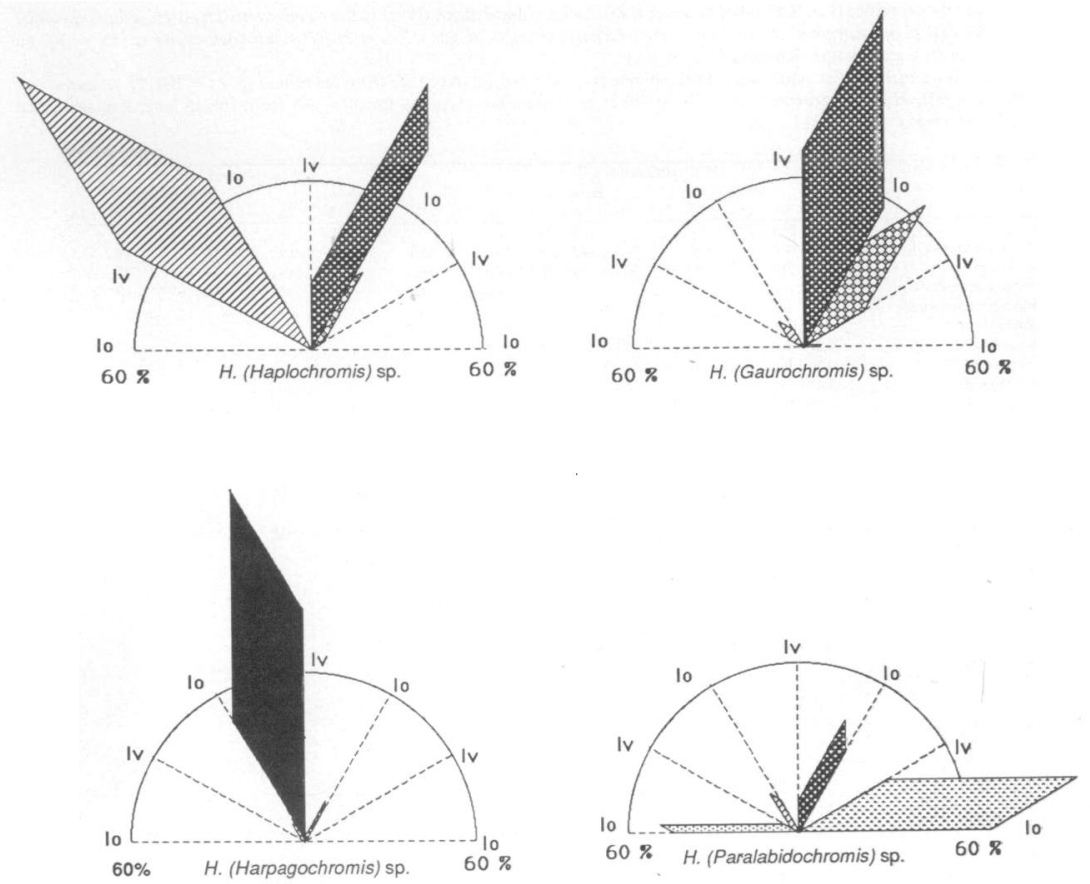

$60 \%$
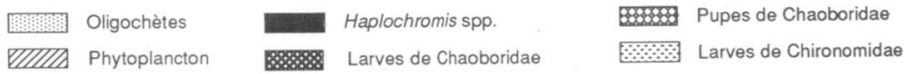

Fig. 2. Régime alimentaire de $H$. (Haplochromis) sp., H. (Gaurochromis) sp., H. (Harpagochromis) sp. et H. (Paralabidochromis) sp. au lac Ihema (surfaces des indices alimentaires IA).

Fig. 2. Food of $H$. (Haplochromis) sp., H. (Gaurochromis) sp., H. (Harpagochromis) sp. and H. (Paralabidochromis) sp. from Ihema lake (surface of feeding indices IA).

Les individus de taille supérieure à $88 \mathrm{~mm}$, montrent une tendance plus marquée dans l'absorption des pupes que des larves de Chaoborus (Tableau II). Le phytoplancton est moins absorbé par les grands individus (Iv de $47 \%$ inférieur) ainsi que le zooplancton qui ne représente qu'une part infime (Iv $\leqslant$ à $0,1 \%$ ) pour chaque catégorie de taille.

Les femelles de $H$. (Gaurochromis) consomment un volume d'insectes légèrement plus important au total que les mâles, cependant on constate une 
différence au niveau du type de proies : les femelles consomment les Chaoborus surtout sous forme de larves (Iv 2 fois supérieur aux mâles) tandis que les mâles examinés consomment les Chaoborus surtout sous formes de pupes (Iv 2,7 fois supérieur aux femelles (Tableau III).

H. (Gaurochromis) sp. présente deux périodes de prise alimentaire (Fig. 3). Il commence à se nourrir vers trois heures du matin jusqu'à l'aube. Il s'ensuit une courte période de digestion puis il reprend son alimentation jusqu'à 16 heures pour ensuite digérer le reste de la journée et une grande partie de la nuit. Son activité locomotrice reste plus ou moins constante durant tout le cycle, excepté à l'aube où ses déplacements sont très intenses.

\subsection{H. (Harpagochromis) sp.}

L'analyse du contenu stomacal de 70 Harpagochromis, de longueur totale comprise entre 68 et $215 \mathrm{~mm}$, a permis de dénombrer une moyenne de 18,2 proies par estomac. La longueur relative de l'intestin, mesurée sur 20 individus, est de $1,74 \pm 0,1$.
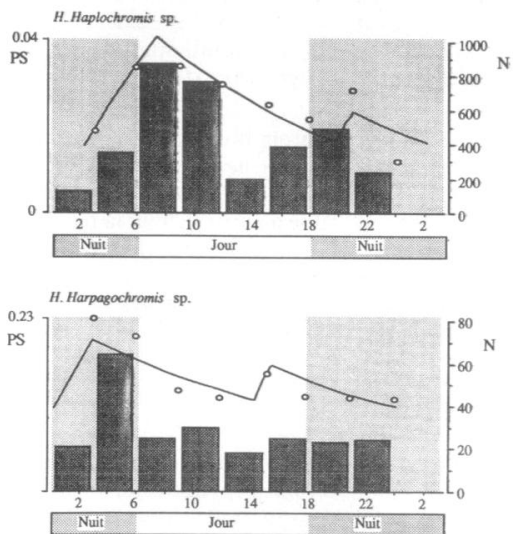

Nombre d'individus capturés $(\mathrm{N})$
Les poissons constituent son alimentation principale (Fig. 2). La catégorie alimentaire la plus importante est constituée d'especes du genre Haplochromis (Iab $=3,2 \% ; \mathrm{IV}=82,5 ; \mathrm{Io}=48,6 \%$ et $\mathrm{IA}=40$ ) suivi des espèces appartenant au genre Tilapia (Iab $=0,47 \% ; \mathrm{IV}=4,4 \%$; Io $=8,57 \% ; \mathrm{IA}=0,4)$. La présence d'œufs n'a été observée que dans les estomacs des femelles. Ceci s'explique par l'absorption, probablement accidentelle lors de la pêche, des œufs de cette espèce à incubation buccale (Tableau I).

La taille des $H$. (Harpagochromis) sp. influence fortement son régime alimentaire (tableau II). En effet, les individus de la classe de taille I (68 à $100 \mathrm{~mm}, \mathrm{n}=17$ ) présentent un indice alimentaire de 6,5 pour les Microcystis aeruginosa, 8,27 pour les Chaoboridae (larves et pupes) et seulement 0,6 pour les Haplochromis spp. alors que la classe de taille II (106 à $215 \mathrm{~mm}, \mathrm{n}=53$ ) n'a qu'un indice alimentaire de 0,006 pour Microcystis aeruginosa et 0,011 pour les Chaoboridae (larves et pupes) mais de 52,0 pour les Haplochromis sp. On peut donc observer un changement radical du régime alimentaire
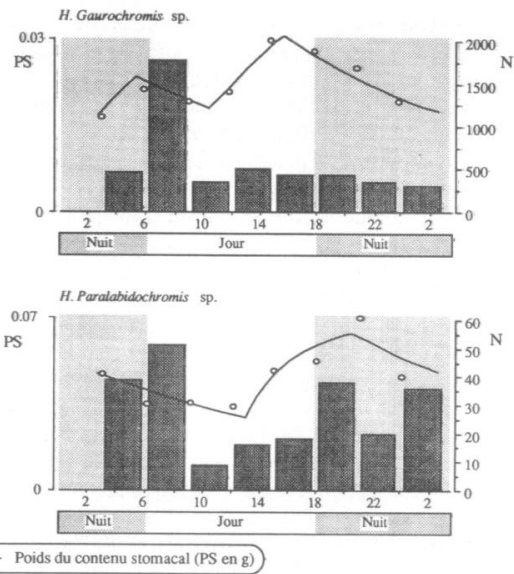

Fig. 3. Représentation du rythme nychtéméral et d'activité locomoteur d'H. (Haplochromis) sp., H. (Gaurochromis) sp., H. (Harpagochromis) sp. et $H$. (Paralabidochromis) sp. au lac Ihema lors de 5 cycles de 24 heures.

Fig. 3. Diel rhythm of locomotory activity of $H$. (Haplochromis) sp., $H$. (Gaurochromis) sp., H. (Harpagochromis) sp. et $H$. (Paralabidochromis) sp. from Ihema lake during 5 cycles of $24 \mathrm{~h}$. 
en fonction de la taille avec pour les jeunes individus ( $\mathrm{Lt}<100 \mathrm{~mm}$ ) une ingestion d'algues et de larves d'insectes principalement tandis que les individus plus âgés (> $106 \mathrm{~mm}$ ) sont ichtyophages.

Le régime alimentaire de $H$. (Harpagochromis) sp. varie peu en fonction des sexes. On note cependant la présence de phytoplancton (Microcystis aeruginosa), et de crustacé (Caridina sp.) uniquement chez les femelles qui présentent donc un régime plus diversifié que les mâles. La présence d'œufs dans les estomacs de ces dernières est probablement le fait du stress lors de la capture qui provoque chez cellesci l'absorption accidentelle des œufs incubés buccalement.

H. (Harpagochromis) sp. présente aussi deux périodes de prise alimentaire (Fig. 3). Il s'alimente une première fois de minuit à $\mathbf{4}$ heures du matin. Il s'ensuit une longue période de digestion puis semble se nourrir à nouveau entre 15 et 16 heures. Hormis la période allant de 3 à 6 heures du matin, $H$. (Harpagochromis) sp. ne présente pas d'activité intense de déplacement.

\subsection{H. (Paralabidochromis) sp.}

L'analyse des contenus alimentaires de cette espèce n'a pu être effectuée que pour 30 individus de longueur totale comprise entre 68 et $128 \mathrm{~mm}$. La longueur relative de l'intestin, mesurée sur 13 individus, est de $2,56 \pm 0,3$. Nous avons pu identifier une moyenne de 43,6 proies par estomac. Le régime alimentaire de cette espèce est plus diversifié que celui des précédentes tout en restant principalement insectivore (Fig. 2). 11 est constitué de larves d'insectes (Chironomidae : Iv $=36,4 \%$; Io $=66,7 \%$ et $\mathrm{IA}=24,2$; Chaoboridae $\mathrm{Iv}=11,4$; Io $=$ $33,3 \%$ et $\mathrm{IA}=3,8)$, d'Oligochètes (Iv $=6,4 \%$; lo $=43,3 \%$ et $\mathrm{IA}=2,8$ ) et, dans une moindre mesure, de phytoplancton (Microcystis aeruginosa) (Iv $=3,7 \% ;$ Io $=13,3 \%$ et IA $=0,5$ ) (Tableau I). Les autres proies semblent être des prises accidentelles. On remarquera l'occurrence élevée de sable et de macrophytes qui s'explique par une alimentation en zone côtière. De plus l'absorption d'œufs est particulièrement élevée ( $\mathrm{Iv}=\mathbf{4 0} \% \mathbf{0})$ mais toujours effectuée par les femelles. Ceci montre qu'il s'agit encore d'une ingestion probablement accidentelle lors de la capture des femelles incubantes. Le spectre alimentaire (Fig. 2) montre clairement la préférence de $H$. (Paralabidochromis) sp. pour la consommation des larves de Chironomidae, des pupes de Chaoboridae et même des oligochètes.

Pour H. (Paralabidochromis) sp. aussi un changement alimentaire est observé en fonction de la taille (Tableau II). Deux régimes correspondant à deux classes de tailles ont pu être différenciés. La classe 1 ( $\mathrm{Lt}=68$ à $85 \mathrm{~mm}, \mathrm{n}=9$ ) montre une occurrence en macrophytes (Io $=22,2 \%$ ) et en sable (lo $=11,1 \%$ ) bien plus faible que celle de la deuxième classe $(\mathrm{Lt}=88$ à $128 \mathrm{~mm}, \mathrm{n}=21)$ qui présente une occurrence en macrophytes de $81 \%$ et en sable de $66,7 \%$. En outre, les individus de la classe de taille 1 ont une alimentation moins diversifiée, n'ingérant principalement que des larves d'insectes Chaoboridae (IA $=32,9$ ) et du phytoplancton : algues Microcystis aeruginosa (IA $=0,26$ ). Nous observons donc que les jeunes $H$. (Paralabidochromis) sp. sont surtout entomophages alors que les individus plus vieux sont plutôt omnivores.

Il semble exister une différence importante entre l'alimentation des mâles et femelles de $H$. (Paralabidochromis) $\mathrm{sp}$. au niveau des insectes ingérés (Tableau III). Les larves de Chironomidae, par exemple, sont consommées en quantité nettement moins importante par les femelles ( $\mathrm{Iv}=\mathbf{2 8 , 6 \%}$ ) que par les mâles (Iv $=89,2 \%$ ). Les femelles de $\boldsymbol{H}$. (Paralabidochromis) sp. consomment une plus grande quantité d'oligochètes ( $\mathrm{IA}=3,1$ ) que les mâles (IA $=0,9)$.

A l'encontre des trois précédentes espèces, $\boldsymbol{H}$. (Paralabidochromis) sp. ne présente qu'un seul moment de prise alimentaire qui se situe entre 12 et 20 heures (Fig. 3). Il se déplace surtout la nuit, plus précisément en début de nuit (entre 18 et 21 heures) et dans la deuxième moitié de la nuit jusqu'en début de journée (de 24 à 9 heures). Après l'aube, ses déplacements se font rares.

\section{Discussion}

Nous avons pu constater dans le régime alimentaire d'H. (Haplochromis) sp. une nette dominance des algues coloniales suivies des larves de Chaoboridae. H. (Haplochromis) sp. pourrait donc être classé comme principalement pilytoplanctonophage et secondairement entomophage. Cependant, nos observations indiquent que les larves de Chaoboridae constituent une proie d'importance. En effet, malgré un pourcentage volumétrique moins élevé 
que pour le phytoplancton, l'occurrence des larves d'insectes Chaoboridae est très supérieure (Io = $79,6 \%$ ) par rapport à celle des algues Microcystis aeruginosa $(\mathrm{Io}=40,1 \%)$ ). Ceci pourrait refléter une certaine préférence de $H$. (Haplochromis) sp. pour les insectes Chaoboridae ou du moins, un besoin alimentaire complémentaire. Certaines données peuvent confirmer cette théorie. D'une part, la longueur relative de l'intestin (LRI) de $\boldsymbol{H}$. (Haplochromis) sp. est de $1,7 \pm 0,09$. Cette valeur se situe légèrement au-dessus des espèces carnivores voraces (LRI $=0,5$ à 1,5) et bien au-dessous des espèces omnivores (LRI $=2,5$ à 3,8 ) et phytophages ou algophages (LRI $\geqslant$ 3,5) (Al Hussaini 1947, Weatherley et al. 1987, Ulyel et al. 1990). D'autre part, une analyse succincte du contenu de l'intestin nous a révélé que les larves d'insectes Chaoboridae y sont mieux broyées et qu'à l'extrémité postérieure du tube digestif, les algues Microcystis aeruginosa restent entières, et présentent une occurrence plus importante. On peut considérer cela comme une meilleure digestibilité des larves de Chaoboridae. Enfin, l'étude du rythme nycthéméral de prise alimentaire de $H$. (Haplochromis) sp. a montré que cette espèce se nourrit deux fois par jour. Une petite prise d'alimentation se fait au début de la nuit et une plus importante en fin de nuit. Nous pensons que ce rythme d'alimentation est dicté par le rythme circadien des larves de Chaoboridae. Ces dernières remontent lentement et massivement en surface dès la tombée de la nuit. C'est à ce moment que commence la première prise d'alimentation d' $H$. (Haplochromis) sp. et que son activité est assez importante. C'est aussi à cette période qu'il se fait capturer presqu' exclusivement par les filets de surface. Ce dernier phénomène s'observe pendant toute la nuit, alors qu'en journée, il se maille plutôt dans les filets de fond. En fin de nuit, lorsque les larves de Chaoboridae redescendent au fond du lac, $H$. (Haplochromis) sp. se nourrit une nouvelle fois jusqu'à la levée du jour, puis occupe, pendant toute la journée, le fond et $y$ digère. Cette similitude de comportement migratoire journalier entre les larves d'insecte et $\boldsymbol{H}$. (Haplochromis) sp. nous amène à penser que les larves de Chaoboridae constituent la proie préférentielle d' $H$. (Haplochromis) sp. et que ce dernier cherche activement cette proie pour ses besoins énergétiques. Dès lors, l'importance des amas de Microcystis aeruginosa dans son alimentation proviendrait soit d'une ingestion accidentelle des algues lorsque le poisson se nourrit des larves de Chaoborus sp. soit que ces algues représentent tout de même un apport énergétique complémentaire.

Toutes ces observations nous amènent à caractériser $H$. (Haplochromis) sp. comme étant un poisson à régime alimentaire limité. Ses proies principales sont, par ordre d'importance, les Chaoborus sp. et l'algue Microcystis aeruginosa.

L'alimentation de $H$. (Gaurochromis) sp. dépend aussi de l'ingestion des larves d'insectes Chaoboridae mais de manière plus importante. A l'inverse d'H. (Haplochromis) sp., H. (Gaurochromis) sp. capture ses proies enfouies pendant la journée dans la vase du lac. Les moments de prise alimentaire sont aussi différents. Un faible pic d'alimentation est observé juste avant l'aube, moment où la prédation se fait vers la surface lorsque les larves de Chaoborus sont encore dans la colonne d'eau pour se nourrir de zooplanton (la prédation semble cependant peu importante à ce moment). La deuxième période d'alimentation, beaucoup plus importante, démarre en pleine journée, juste avant midi. A ce moment, les Chaoborus ingérés par $H$. (Gaurochromis) sp. ne peuvent provenir que de la vase du fond du lac, ce qui montre bien la recherche active faite par $H$. (Gaurochromis) sp. pour trouver sa proie. Notons aussi que les captures, pendant la journée, sont réalisées presqu'exclusivement par les filets maillants disposés sur le fond du lac. Les filets de surface n'attrapent qu'occasionnellement cette espèce, sauf de 21 à 6 heures.

Pour $H$. (Harpagochromis) sp., on a observé un régime alimentaire principalement piscivore avec une prédominance pour l'ingestion des Haplochromis spp. C'est chez cette espèce que l'on constate la plus forte variation du régime alimentaire en fonction de la taille ce qui semble normal pour un piscivore. Le caractère ichtyophage ne s'exprime nettement qu'à partir d'une taille supérieure à $106 \mathrm{~mm}$. Au delà de cette taille, son rythme d'alimentation est aussi caractérisé par deux périodes de prédation, dont la plus intense se situe en fin de nuit.

C'est en zone côtière que l'on capture principalement $H$. (Paralabidochromis) sp. Il est dès lors nomal de constater une occurrence élevée en sable et en macrophytes. Son régime alimentaire est plus diversifié que les autres espèces et se compose surtout de Chironomidae, de Chaoboridae et d'Oligochètes). Pourtant, la longueur relative de l'intestin 
(calculée sur 13 individus) est de 2,56 $\pm 0,3$; ce qui correspond aux valeurs observées en général pour les espèces omnivores (LRI : 2,5 à 3,8). On peut donc supposer que, avec leur important pourcentage d'occurrence, les macrophytes constituent un aliment d'importance assez élevée délibérément choisi par cette espèce.

Les différences d'alimentation entre les sexes sont probablement biaisées par l'observation accidentelle des œufs incubés (Iv $=45,9 \%$ ). Si l'on fait abstraction de ceux-ci, on obtient certes une différence entre les sexes mais moins prononcée, en ce qui concerne les Chironomidae ( $\mathrm{Iv}=52,7 \%$ pour les femelles et $89,2 \%$ pour les mâles). Par contre, les femelles montrent une préférence plus marquée pour les larves de Chaoboridae dont l'indice volumétrique ( $\mathrm{IV}=24,5 \%)$ est 33 fois supérieur à celui des mâles. Ces résultats devront cependant être confirmés car un nombre peu élevé d'estomacs de mâles de $H$. (Paralabidochromis) sp. a pu être analysé. On peut cependant penser qu'étant incubatrices buccales, les femelles tardent plus à se nourrir pendant la journée et qu'elles profitent ainsi, en début de nuit, de l'apport massif et aisément capturable des larves de Chaoboridae.

En ce qui concerne maintenant uniquement les rythmes d'activité locomoteurs des quatre espèces nous avons pu constater que chaque taxa possède son propre rythme. $H$. (Haplochromis) sp. présente deux pics d'activité : durant la matinée et en début de soirée. Pour H. (Gaurochromis) sp. et $H$. (Harpagochromis) sp. nous n'avons observé qu'une seule période d'activité, très intense, se situant, respectivement en début de matinée et en fin de nuit. Enfin, H. (Paralabidochromis) sp. circule pendant la nuit jusqu'en début de matinée.

\section{Conclusion}

On observe pour chaque espèce une spécialisation alimentaire marquée permettant d'éviter ainsi une compétition trophique trop importante et ce, malgré l'utilisation des larves de Chaoboridae par trois des quatre espèces étudiées. Pour cette ressource alimentaire commune, il apparaît qu'elle est prélevée à des moments et des lieux différents. H. (Haplochromis) sp. mange ces larves en zone pélagique, de préférence près de la surface, lorsqu'elles y ont migré pendant la nuit (ce poisson complète vraisemblablement son régime alimentaire en absorbant aussi des amas de Microcystis aeruginosa). Pour $H$. (Gaurochromis) sp. ces larves constituent l'apport principal de son alimentation. Il les consomme aussi en zone pélagique, mais plutôt au fond du lac lorsqu'elles redescendent et s'enfouissent dans la vase en fin de nuit, lieu où $H$. (Gaurochromis) sp. sait aller les chercher et s'en nourrir même en pleine journée. Enfin, H. (Paralabidochromis) sp. les ingère, en quantité moins importante, en zone côtière et complète son alimentation par d'autres proies.

La dernière espèce étudiée est $H$. (Harpagochromis) sp. qui, au delà d'une taille de $106 \mathrm{~mm}$, est un ichtyophage strict.

Nous sommes donc, au lac Ihema, en présence de taxa certainement phylogénétiquement proches et qui ont réussi, au cours de leur évolution, à réduire la compétition alimentaire en développant des stratégies spatio-temporelles différentes et en occupant finalement 4 niches écologiques distinctes. De telles spécialisations ont été notées également pour les nombreux Haplochromis sp. du lac Victoria (Witte 1981, Witte \& Goudswaard 1984). Cette diversité semble être au lac Victoria ou Nabugabo, le résultat d'un isolement antérieur suivi d'une réunification après spéciation divergente (Greenwood 1965). La spécialisation alimentaire des espèces du groupe Haplochromis du lac Ihema pourrait provenir d'une situation similaire à moins que ces espèces ne soient issues elles-mêmes des espèces du lac Victoria présentant des régimes alimentaires déjà différenciés. Leur migration vers l'Akagera aurait pu avoir lieu notamment lors de l'élévation du niveau du lac Victoria qui aurait envahi la vallée de l'Akagera il y a moins de 60000 ans (Fryer \& Iles 1972, Beadle 1981, Witte 1981 et 1987).

\section{Remerciements}

Cette étude a pu être réalisée grâce au soutien financier du Centre de Coopération de Développement (CECODEL) de l'Université de Liège et à l'Administration Générale de la Coopération au Développement (A.G.C.D.) en Belgique. Nous leur en sommes très vivement reconnaissants.

L'Office Rwandais du Tourisme et des Pares Nationaux (O.R.T.P.N.) nous a autorisé à travailler au lac Ihema dans le Parc National de l'Akagera. Que son Directeur e son personnel reçoivent tous mos remerciements. 
Notre gratitude s'adresse particulièrement au Directeur de la Pêcherie Ihema R. Bambujimugisha (t) trop tố disparu. Finalement, nous désirons remercier vivement Madame S. Coppin, Monsieur P. Degee et Madame B. Dumont du CECODEL de l'Université de Liège pour leur intérêt et leur soutien apprécié.

\section{Travaux cités}

Al-Hussaini A.H. 1947. - The anatomy and histology of the alimentary tract of the plankton-feeder, Atherina forskali Rüpp. J. Morph. Philad., 80, 251-286.

Beadle L.C. 1981. - The inland waters of tropical Africa. An introduction to tropical limnology. Londre a New York, 2nde édition, Longman ed. : $475 \mathrm{p}$.

Daget J., Gosse J.P., Teugels G.G. \& Thys Van Den Audenaerrden D. 1991. - Catalogue des poissons d'eau douce d'Afrique. Cloffa 4, Paris, ISNB MRAC ORSTOM : $740 \mathrm{p}$.

Fryer G. \& Iles T.D. 1992. - The cichlid fishes of the great lakes of Africa. Edinburgh, Oliver \& Boyd : 641 p.

Greenwood P.H. 1965. - The cichlid fishes of lake Nabugabo, Uganda. Bull. Br. Mus. Nat. Hist. (Zool.), 12, 315-357.

Greethwood P.H. 1979. - Toward a phyletic classification of the " genus " Haplochromis (Pisces, Cichlidae) and related taxa. Part. 1. Bull. Br. Mus. Nat. Hist. (Zool.), 35, 265-322.

Greenwood P.H. 1980. - Toward a phyletic classification of the « genus » Haplochromis (Pisces, Cichlidae) and related taxa. Part 2 : the species from Lakes Victoria, Nabugabo, Edward, George and Kivu. Bull, Br. Mus. Nat. Hist. (Zool.), 39, 1-101.

Jarre A., Palomares M.L., Soriano M.L., Sambilay V.C. \& Pauly D. 1990 . - Maxims : a computer for estimating the food consumption of fishes from diet stomach contents data and population parameters. User's manual : Test version. ICLARM software 4: $27 \mathrm{p}$.

Kiss R. 1976. - Etude hydrobiologique des lacs de l'Akagera Moyenne. Inst. Nat. Rech. Sc., Rwanda, 16: 167 p.

Lauzanne L. 1975. - La sélection des proies chez trois poissons malacophages du lac Tchad. Cah. ORSTOM, sér. hydrobiol. 9, 1, 3-7.
Lauzanne L. 1976. - Régime alimentairc et rclations trophiques des poissons du lac Tchad. Cah. ORSTOM, séri. hydrobiol. $10,4,267-310$

Micha J.C. \& Frank V. 1991. - Ressources et caractéristiques piscicoles des lacs rwandais (Bassins nilotiques). Bull. Séanc. Acad. r. Sci, Outre-Mer, 37, 3, sous presse.

Plisnier P.-D. 1990. - Ecologie comparée el exploitation rationnelle de deux populations d'Haplochromis spp (Teleostei, Cichlidae) des lacs Ihema et Muhazi (Rwanda). These de Dr. Sciences Agronomiques, UCL, Louvain-La-Neuve : 324 p.

Plisnier P.-D., Micha J. C. \& Frank V. 1988. - Biologie et exploitation des poissons du lac lhema (Bassin Akagera, Rwanda). Namur, Presses Universitaires de Namur : 212 p.

Ulyel A.P., Ollevier R., Ceusters R.\& Thys Van Den Audenaerden D. 1990 . - Régime alimentaire des Haplochromis (Teleostei : Cichlidae) du lac Kivu en Afrique. I. Relations trophiques interspécifiques. Belg. J. Zool. 1202 : 143-155.

Vollenweider R.A. 1969. - A manual on methods for mesuring primary production in aquatic environments including a chapter on bacteria. Vollenweider. Oxford. R.A. ed. : 229 p.

Weatherley A.H., Gill H.S. \& Casselman J.M. 1987. - The biology of fish growth. London, Academic Press : $443 \mathrm{p}$.

Witte F. 1981. - Initial results of the ecological survey of the Haplochromis Cichlid Fishes from the Mwanza Gulf of Lake Victoria (Tanzania) : breeding patterns, trophic and species distribution Neth. J. Zool., 31, 175-202.

Witte F. 1987. - From form to fishery. An Ecological and taxonomical contribution to morphology and fishery of lake Victoria Cjchlids. Thèse Doctorat Univ. Leiden, Netherlands : $140 \mathrm{p}$.

Witte F. \& Goudswaard P.C. 1984. - Reports from the Haplochromis Ecology Survey Team (HEST) operating in the Mwanza area of Lake Victoria-Prospects of the haplochromine fishery in southern Lake Victoria Report presented as the third meeting of the FAO CIFA-Subcommittee on Lake Victoria at Jinja (Uganda), October 1-5, 1984, $14 \mathrm{p}$. 